\title{
Clustering through postinhibitory rebound in synaptically coupled neurons
}

\author{
D. T. W. Chik, ${ }^{1}$ S. Coombes, ${ }^{2}$ and Z. D. Wang ${ }^{1}$ \\ ${ }^{1}$ Department of Physics, The University of Hong Kong, Hong Kong, China \\ ${ }^{2}$ School of Mathematical Sciences, University of Nottingham, Nottingham NG7 2RD, United Kingdom
}

(Received 1 March 2004; published 15 July 2004)

\begin{abstract}
Postinhibitory rebound is a nonlinear phenomenon present in a variety of nerve cells. Following a period of hyperpolarization this effect allows a neuron to fire a spike or packet of spikes before returning to rest. It is an important mechanism underlying central pattern generation for heartbeat, swimming and other motor patterns in many neuronal systems. In this paper we consider how networks of neurons, which do not intrinsically oscillate, may make use of inhibitory synaptic connections to generate large scale coherent rhythms in the form of cluster states. We distinguish between two cases (i) where the rebound mechanism is due to anode break excitation and (ii) where rebound is due to a slow T-type calcium current. In the former case we use a geometric analysis of a McKean-type model to obtain expressions for the number of clusters in terms of the speed and strength of synaptic coupling. Results are found to be in good qualitative agreement with numerical simulations of the more detailed Hodgkin-Huxley model. In the second case we consider a particular firing rate model of a neuron with a slow calcium current that admits to an exact analysis. Once again existence regions for cluster states are explicitly calculated. Both mechanisms are shown to prefer globally synchronous states for slow synapses as long as the strength of coupling is sufficiently large. With a decrease in the duration of synaptic inhibition both systems are found to break into clusters. A major difference between the two mechanisms for cluster generation is that anode break excitation can support clusters with several groups, while slow T-type calcium currents predominantly give rise to clusters of just two (antisynchronous) populations.
\end{abstract}

DOI: 10.1103/PhysRevE.70.011908

PACS number(s): 87.18.Sn, 87.19.La

\section{INTRODUCTION}

Recordings from nerve cells demonstrate that if the potential at the cell body is increased above a certain threshold value, then a pulselike signal can be initiated along the outgoing axon. In addition to the action potential, one other important nonlinear phenomenon in a variety of nerve cells is post inhibitory rebound (PIR). Here the excitability of the neuron is enhanced temporarily following a period of voltage depression (hyperpolarization). As a result the neuron may fire a single spike or a packet of spikes (i.e., it may burst). Post inhibitory rebound has been shown experimentally to play an important role in central pattern generating networks that produce rhythmic output [1-4], as well as providing a neural mechanism for the extraction of temporal cues in human speech [5]. The theoretical importance of post inhibitory rebound for central pattern generation in networks of nonoscillatory neurons was perhaps first recognized by Perkel and Mulloney [6], although dates back to work by Brown [7] on so-called half-center oscillators. In certain nerve cells, such as those of the medicinal leech, the ionic mechanism for post inhibitory rebound has been uncovered [8,9]. It is important to note, however, that many excitable models of neural membrane, such as the Hodgkin-Huxley and FitzHughNagumo model, are known to exhibit a form of post inhibitory rebound that is more properly called anode-break excitation. Thus it becomes interesting to distinguish between mechanisms for rebound based upon the basic mechanism of anode break excitation (common to many excitable neuron models) and novel ionic currents that do not form part of the make-up of minimal models of excitable membrane. Specifically we are interested in the slow T-type calcium current known to underly bursting behavior in single neurons upon release from inhibition. This particular current is known to play an important role within the context of thalamocortical oscillations $[10,11]$.

The focus of this paper will be on the existence of phaseclustered states as a function of the speed and strength of inhibitory synaptic interaction in networks of globally coupled neurons. Such states are a collection of subpopulations within a network each of which consists of a fully phase-synchronized set of neurons. Note that we are concerned with the strong coupling of elements that do not intrinsically oscillate. As such this work is complementary to previous important studies of globally coupled phaseoscillators, such as by Golomb et al. [12], Hansel et al. [13], and Okuda [14], relevant for networks of weakly interacting oscillators.

In Sec. II we consider the Hodgkin-Huxley model of an excitable membrane and its reduction to a planar dynamical system. This more easily allows us to describe the phenomenon of anode break excitation using geometric notions. The equations of motion of the reduced Hodgkin-Huxley model are then approximated in a piecewise linear fashion to obtain a single neuron model of McKean-type [15]. Under the assumption of a separation of time scales for the voltage and recovery variables of this modified McKean model we are able to exactly quantify the PIR response of the neuron to an inhibitory step input. This analysis forms the basis for a subsequent network study with global inhibitory synaptic connections of simple on/off type. Analytical expressions for the existence of phase-clustered states are obtained in the singular limit and shown to be in good qualitative agreement with simulations of a Hodgkin-Huxley network.

In Sec. III we consider a spiking neuron model, possessing a slow T-type calcium current, that can support a rebound 
spike burst in response to an inhibitory synaptic current. For slow synaptic inhibition we are able to formulate network dynamics in terms of a firing rate model. An exact analysis of this model is possible for a Heaviside firing rate function and is used to calculate the regions in parameter space where cluster states exist. In comparison to Sec. II we do not explicitly exploit any underlying geometric features of the dynamics and are able to consider a more general class of synaptic response functions.

Finally, in Sec. IV we discuss the similarities and differences between cluster states generated by the two mechanisms discussed in this paper.

\section{ANODE BREAK EXCITATION}

In the Hodgkin-Huxley model of excitable nerve tissue the membrane current arises mainly through the conduction of sodium and potassium ions through voltage dependent channels in the membrane [16]. The contribution from other ionic currents is assumed to obey Ohm's law. In fact the Hodgkin-Huxley dynamics is considered to be a function of membrane potential $v$ and three time and voltage dependent conductance variables $m, n$, and $h$ :

$$
\begin{aligned}
\mu \frac{\mathrm{d} v}{\mathrm{~d} t} & =F(v, m, n, h) \\
& \equiv-g_{L}\left(v-v_{L}\right)-g_{\mathrm{K}} n^{4}\left(v-v_{K}\right)-g_{\mathrm{Na}} h m^{3}\left(v-v_{\mathrm{Na}}\right)+I .
\end{aligned}
$$

Here, $\mu$ is the membrane capacitance, $g_{\mathrm{K}}, g_{\mathrm{Na}}$, and $g_{L}$ are constants and $V_{L}, V_{\mathrm{K}}$, and $V_{\mathrm{Na}}$ represent the constant membrane reversal potentials associated with the leakage, potassium, and sodium channels, respectively. $I$ is an externally injected current. The conductance variables $m, n$, and $h$ take values between 0 and 1 and approach the asymptotic values $m_{\infty}(v), n_{\infty}(v)$, and $h_{\infty}(v)$ with time constants $\tau_{m}(v), \tau_{n}(v)$, and $\tau_{h}(v)$, respectively. Summarizing, we have that

$$
\tau_{X}(v) \frac{\mathrm{d} X}{\mathrm{~d} t}=X_{\infty}(v)-X, \quad X \in\{m, n, h\} .
$$

The six functions $\tau_{X}(v)$ and $X_{\infty}(v), X \in\{m, n, h\}$, are obtained from fits with experimental data (given in the Appendix).

A systematic approach for reducing the dimension of Hodgkin-Huxley-type models has been proposed by Abbott [17], called the method of equivalent potentials. We may use this approach to obtain a reduced two-dimensional version of the Hodgkin-Huxley model that can be readily investigated with the tools of phase-plane analysis and geometry. In essence this approach makes use of the fact that $\tau_{m}(v)$ is small for all $v$ so that the variable $m$ rapidly approaches its equilibrium value $m_{\infty}(v)$. Moreover, the equations for $h$ and $n$ have similar time-courses, so that they may be slaved together via a so-called equivalent potential, $w$. The result of this procedure is a two dimensional model with membrane current $f(v, w)=F\left(v, m_{\infty}(v), n_{\infty}(w), h_{\infty}(w)\right)$, such that

$$
\mu \frac{\mathrm{d} v}{\mathrm{~d} t}=f(v, w)+I, \quad \frac{\mathrm{d} w}{\mathrm{~d} t}=g(v, w),
$$

where

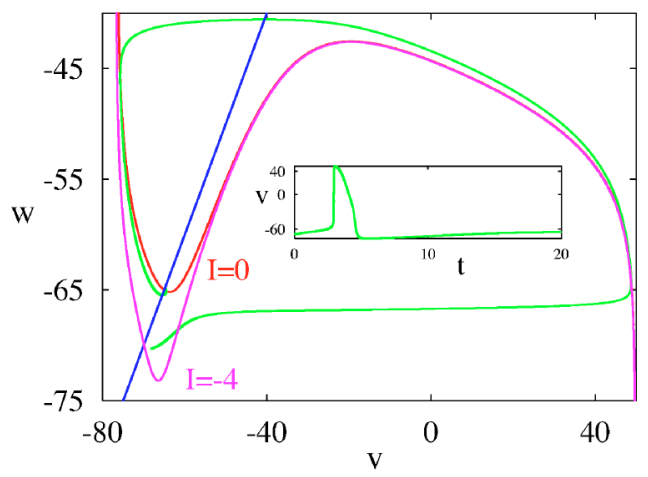

FIG. 1. (Color online) The phase-plane for the reduced Hodgkin-Huxley model obtained by the method of equivalent potentials. The straight diagonal line is the $w$ nullcline, $w=v$, while the two "cubic" curves are the $v$ nullclines with $I=0$ and $I=-4$. The inset shows the rebound spike that arises when the fixed point with $I=-4$ is abruptly removed, by setting $I=0$.

$$
g(v, w)=\frac{\frac{\partial F}{\partial h}\left[\frac{h_{\infty}(v)-h_{\infty}(w)}{\tau_{h}(v)}\right]+\frac{\partial F}{\partial n}\left[\frac{n_{\infty}(v)-n_{\infty}(w)}{\tau_{n}(v)}\right]}{\frac{\partial f}{\partial h_{\infty}} \frac{\mathrm{d} h_{\infty}(w)}{\mathrm{d} w}+\frac{\partial f}{\partial n_{\infty}} \frac{\mathrm{d} n_{\infty}(w)}{\mathrm{d} w}},
$$

and $\partial F / \partial h$ and $\partial F / \partial n$ are evaluated at $h=h_{\infty}(w)$ and $n$ $=n_{\infty}(w)$. The variable $v$ corresponds to a membrane potential while $w$ is associated with the refractory properties of a neuron. One natural consequence of this reduction is that the nullcline for $w$ (defined by $\dot{w}=0$ ) is the straight line $w=v$. The voltage nullcline has a more cubic shape, as expected for a model of excitable membrane. A plot of the phase-plane for this model is given in Fig. 1. It is convenient to discuss the "cubic" nullcline in terms of left, middle and right hand branches. When $I=0$ the fixed point falls on the left hand branch and is stable. With increasing $I$ the fixed point can become unstable and moves on to the middle branch. As it goes unstable (in a Hopf-bifurcation) one sees the appearance of a stable periodic orbit. However, our interest is in the response of the system to inhibitory input. Consider for the moment a negative value of $I$ such that the fixed point moves to a hyperpolarized value (with respect to the case when $I$ $=0$ ). Since this fixed point is also stable it will remain there for all time. However, an abrupt removal of this inhibition leads to a rebound spike: to equilibrate back to the fixed point the system makes a transition to the right-hand branch, as illustrated in Fig. 1, before jumping back to the left-hand branch and relaxing to the fixed point for $I=0$. We see that the systematic reduction of the Hodgkin-Huxley model to the plane is a natural way in which to uncover the geometric mechanism underlying anode break excitation. The mechanism of anode break excitation is clearly dependent on the overall cubic shape of the $v$ nullcline, but is independent of any detailed structure. Hence, further insight is likely to come from a simpler (yet similar) choice of this shape. We note from Fig. 1 that the main effect of an inhibitory drive is to simply reduce the minima of the $v$ nullcline while leaving 


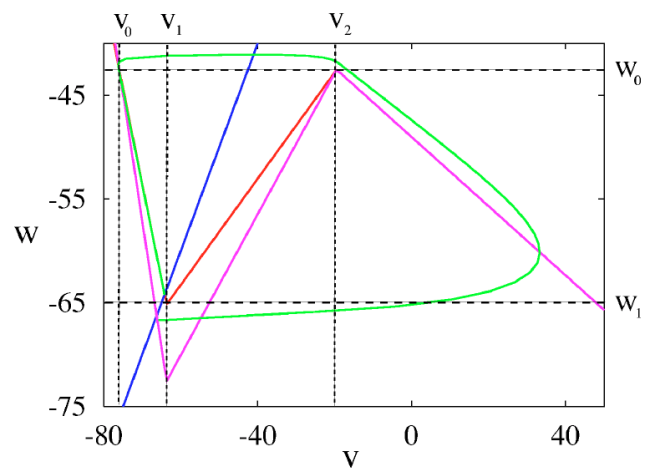

FIG. 2. (Color online) The phase-plane for the modified McKean model, with $v$ nullclines plotted for $I=0$ and $I=-4$. Also shown is a rebound spike created by removing inhibition from the rest state with $I=-4 . \mu=0.01, w(x)=4 \log (1+x / 4.75)$ and other parameters as in Fig. 1.

other parts of the curve relatively unchanged. From the desire to work with a mathematically tractable model that captures the essential features of the Hodgkin-Huxley model we therefore introduce the following choices for $f(v, w)$ and $g(v, w)$ :

$$
\begin{gathered}
f(v, w)+I=f(v ; I)-w+w_{0}, \\
g(v, w)=v-w,
\end{gathered}
$$

where

$$
f(v ; I)= \begin{cases}-\left(v-v_{0}\right) / \beta_{0}(I) & v<v_{1}, \\ \left(v-v_{2}\right) / \beta(I) & v_{1} \leqslant v \leqslant v_{2}, \\ -\left(v-v_{2}\right) / \beta_{1} & v>v_{2} .\end{cases}
$$

Parameters of this model are easily fit to the reduced Hodgkin-Huxley model and have a natural physical interpretation as points of maxima and minima of the $v$ nullcline or gradients on the left, middle, and right branches. This is most easily described with the aid of the plot in Fig. 2. Here we have set $\beta_{1}=3$ and

$$
\beta_{0}(x)=\frac{v_{1}-v_{0}}{w_{0}-w_{1}(x)}, \quad \beta(x)=\frac{v_{2}-v_{1}}{w_{0}-w_{1}(x)},
$$

with $w_{0}=-42.5, \quad w_{1}=-65.2, \quad v_{0}=-76.0, \quad v_{1}=-63.7, \quad v_{2}$ $=-19.5$. The function $w_{1}(x)=w_{1}+w(x)$, describes how much the minima of $v$ is perturbed by a constant current injection. A detailed comparison with the reduced Hodgkin-Huxley model suggests a choice such as $w(x)=4 \log (1+x / 4.75)$ $(x<0)$. However, for the sorts of mechanistic questions we are interested in simpler choices, such as $w(x)=x$, are equally as valid. We call the above the modified McKean model since it has piecewise linear nullclines, as does the original McKean model [15]. However, we take more care in obtaining an approximation of the Hodgkin-Huxley model by fitting to the properties of the reduced Hodgkin-Huxley model.

Mathematical progress in quantifying anode break excitation for this model can be made under the simplifying assumption of fast relaxation which allows one to use some of the tools of geometric singular perturbation theory. The sys- tem has nullclines defined by $w-w_{0}=f(v ; I)$ and $w=v$. The case when the fixed point is such that $v<v_{1}$ is said to define the excitable regime. It is convenient to keep track of which branch of the nonlinear function (7) is playing a role in the dynamics. If the time scale for the $v$ dynamics is fast compared to the time-scale for the $w$ dynamics (i.e., in the limit as $\mu \rightarrow 0)$, then $v$ spends no appreciable time off of the nullclines for $\dot{v}=0$. When $v<v_{1}$ we shall say that the system is on the left-hand branch: $w-w_{0}=\left(v_{0}-v\right) / \beta_{0}(I)$ and when $v>v_{2}$ the system is on the right-hand branch: $w-w_{0}=\left(v_{2}\right.$ $-v) / \beta_{1}$. In this case the slow dynamics takes the form

$$
\dot{w}= \begin{cases}A_{0}(I)-\gamma_{0}(I) w & v<v_{1}, \\ A_{1}-\gamma_{1} w & v>v_{2},\end{cases}
$$

where $\gamma_{0}(x)=1+\beta_{0}(x), \quad \gamma_{1}=1+\beta_{1}, A_{0}(x)=v_{0}+\beta_{0}(x) w_{0}, A_{1}$ $=v_{2}+\beta_{1} w_{0}$ and we have adopted the notation $\dot{w}=\mathrm{d} w / \mathrm{d} t$. Note that a necessary condition for anode break excitation within this model is that the fixed point on the left branch be lower than the minima with $I=0$, i.e., $A_{0}(I) / \gamma_{0}(I)<w_{1}$. Networks of weakly coupled oscillatory McKean neurons have previously been discussed in $[18,19]$. We shall now pursue the case of strong inhibitory coupling and the emergence of network rhythms that can coexist with a stable quiescent network state.

At a synapse presynaptic firing results in the release of neurotransmitters that cause a change in the membrane conductance of the postsynaptic neuron. This postsynaptic current may be written

$$
I(t)=\left(v_{s}-v\right) u(t),
$$

where $v$ is the voltage of the postsynaptic neuron and $v_{s}$ is the membrane reversal potential. The variable $u$ corresponds to the probability that a synaptic receptor channel is in an open conducting state. This probability depends on the presence and concentration of neurotransmitter released by the presynaptic neuron. The sign of $v_{s}$ relative to the steady state resting potential, $v_{\mathrm{ss}}$, determines whether the synapse is excitatory $\left(v_{s}>v_{\mathrm{ss}}\right)$ or inhibitory $\left(v_{s}<v_{\mathrm{ss}}\right)$. In this paper we will regard the post-synaptic conductance as a train of pulses, each one induced by the arrival of a presynaptic action potential at a time $T^{m}(m \in \mathbb{Z})$ :

$$
u(t)=\sum_{m} \eta\left(t-T^{m}\right) .
$$

The arrival times are calculated according to a voltage jump condition in the presynaptic neuron, which we shall take to occur as the neuron makes a transition from the left to right branch of the $v$ nullcline. The shape of the post synaptic conductance is given by the function $\eta(t)(\eta(t)=0, t<0)$. So that simple geometric arguments can be used we shall consider on/off type synapses of the form

$$
\eta(t)=g_{s} \Theta(t) \Theta\left(\alpha^{-1}-t\right) .
$$

Here $\alpha^{-1}$ is the duration of a rectangular pulse, $g_{s}$ its strength and $\Theta(t)$ is a Heaviside step function. We shall also focus on the case of strong inhibition so that $v_{s}-v \approx v_{s}$, (i.e., we drop the effects of shunting). For a globally coupled network of $N$ neurons we consider the natural extension 


$$
I_{i}(t)=-\frac{J}{N} \sum_{j=1}^{N} \sum_{m} \Theta\left(t-T_{j}^{m}\right) \Theta\left(\alpha^{-1}-t+T_{j}^{m}\right),
$$

where $J=-g_{s} v_{s}>0$ and $I_{i}(t)$ is interpreted as the input to the ith neuron, with $i=1, \ldots, N$.

For a globally coupled network the symmetry to permutations implies the existence of a homogeneous solution. This could either be a homogeneous fixed point (HFP), in which all neurons in the system remain at rest or a homogeneous limit cycle (HLC), in which all neurons oscillate synchronously. This latter solution is what we shall refer to as a single cluster state. It is also possible that other cluster states will arise through a process of spontaneous symmetry breaking. The most symmetric cluster states will be ones in which there are $M$ clusters, each consisting of $N / M$ fully synchronized neurons, with a nonzero phase difference between each cluster. We might more properly call this a splay-cluster state as we would expect the phase difference between any two groups to be an integer multiple of $2 \pi / M$. Note that we may also interpret the HLC as a 1-cluster state.

First let us consider the construction of a globally synchronous state (a single cluster). If a cluster is released from inhibition and makes a transition from the left to right branch then inhibition is immediately reinstated. The cluster evolves on the right-hand branch until it jumps back to the left on reaching $w_{0}$. By assumption it is still in the inhibited state and will evolve on the left-hand branch until it is released from inhibition (when the synapse turns off). Hence, the condition for the existence of such a solution is that the value of $w$ at the jump from left to right branches be lower than the minimum value of the $v$ nullcline without inhibition (when $J=0$ ). The period of oscillation is simply the duration of the synapse $\alpha^{-1}$, so that a critical value of $\alpha$ may be defined in terms of the minimum period of oscillation. This minimum period is simply the time spent on the left and right branches when the jumping off point (from left to right branches) is equal to $w_{1}$. Since the dynamics for $w$ is piecewise linear it is a simple matter to calculate these times and obtain a condition on $\alpha$ as $\alpha<1 /\left(\Delta_{L}(-J)+\Delta_{R}\right)$ where

$$
\begin{gathered}
\Delta_{R}=\frac{1}{\gamma_{1}} \log \left(\frac{A_{1}-\gamma_{1} w_{1}}{A_{1}-\gamma_{1} w_{0}}\right), \\
\Delta_{L}(I)=\frac{1}{\gamma_{0}(I)} \log \left(\frac{A_{0}(I)-\gamma_{0}(I) w_{0}}{A_{0}(I)-\gamma_{0}(I) w_{1}}\right) .
\end{gathered}
$$

Now let us turn our attention to an $M$-cluster state. In such a state we may imagine that there is a phase relationship between clusters such that at any given time there is a constant level of inhibition given by $I=-J / M$ (apart from the times of measurezero where transitions occur). To maintain this constant level requires that just before release from inhibition all $M$ clusters evolve on the left-hand branch. A single cluster then evolves on the right hand branch and makes a transition back to the left-hand branch before inhibition terminates. In this way there are again $M$ clusters on the left-hand branch (each feeling an inhibition of $-J / M$ ) when the next cluster makes a transition to the right-hand branch. The total period of oscillation is simply $M$ multiples of the duration of syn-

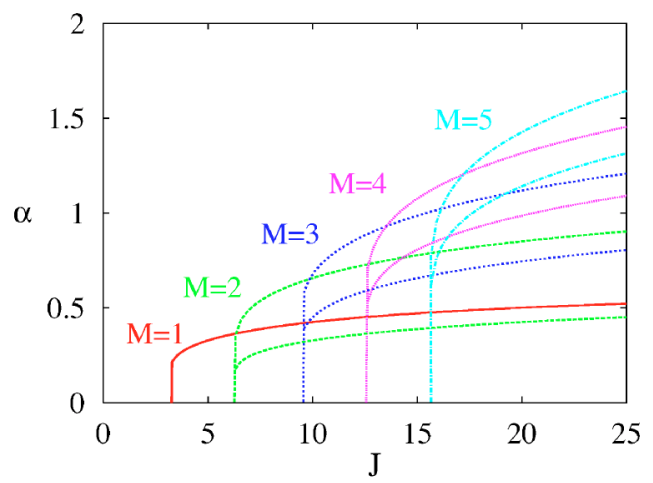

FIG. 3. (Color online) A plot of the critical curves $\alpha(M)$ as a function of $J$ (i.e., synaptic speed vs synaptic strength), defining the regions of existence for $M$-cluster states. Here we choose $w(x)=x$ and all other parameters as in Fig. 2. Note the coexistence of states.

aptic inhibition, which is also equal to the time spent on the left and right branches over a single orbit. Hence, using an identical argument as for the 1-cluster state we obtain a condition on $\alpha=\alpha(M)$ as

$$
\alpha(M)<\frac{M}{\Delta_{L}(-J / M)+\Delta_{R}} .
$$

Moreover, since we have assumed that only one cluster can make the jump from left to right branch we also have that

$$
\alpha(M)>\frac{M-1}{\Delta_{L}(-J / M)+\Delta_{R}} .
$$

In Fig. 3 we plot these critical curves for $M=1, \ldots, 5$ as a function of the strength of inhibition. We see that for small $\alpha$ (slow synapses) there is a critical value of $J$ above which a 1-cluster (HLC) state can be found. Moreover, with increasing $J$ one sees windows of $\alpha$ values where $M$-cluster states can exist. From this figure it is also apparent that there is co-existence of cluster states. For example with $J=7$ we see that it is possible to find a region of $\alpha$ values where there is co-existence of the $M=1$ and $M=2$ states. Moreover with an increase in $J$ and $\alpha$ it is possible to find a region of parameter space where $M=2$ and $M=3$ can coexist. A further increase in $\alpha$ leaves only $M=3$ as a possibility, and with a large enough choice of $\alpha$ no clusters are possible. Note that the HFP is a trivial solution that exists for all $\alpha$ and $J$. Direct numerical simulations of a Hodgkin-Huxley network are found to be consistent with the qualitative predictions of this analysis. Quantitative predictions are not expected as our theory has been developed in the singular limit $(\mu \rightarrow 0)$, which does not hold for the standard parameter set of the Hodgkin Huxley model (given in the Appendix). However, an increasing quantitative agreement between theory and numerics is obtained with an (artificial) decrease in the capacitance of the Hodgkin-Huxley model, as expected. In Fig. 4 we show simulations of $N=120$ Hodgkin-Huxley neurons, illustrating the coexistence of a 1-cluster and 2-cluster state. With an increase in the strength of inhibition $J$ and choosing a faster synapse it is possible to find a coexisting 2-cluster 


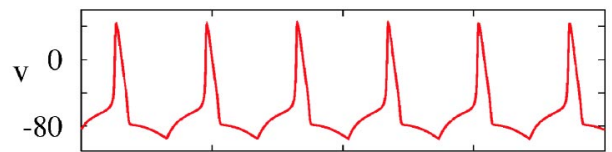

(a) $100 \quad 125 \quad 150 \quad 175 \quad 200$

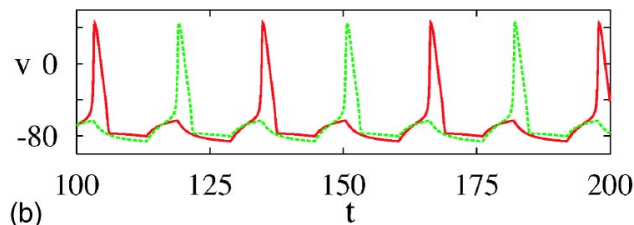

FIG. 4. (Color online) Direct numerical simulations of a network of $N=120$ Hodgkin-Huxley neurons, showing the coexistence of a 1-cluster (top) and a 2-cluster (bottom) state for $\alpha=0.1$ and $J$ $=20$. In the top trace all of the neurons in the globally coupled network synchronize. In the bottom figure (with a different set of initial conditions) the network splits into two equally sized clusters that oscillate in antiphase.

and 3-cluster state, as predicted from the trend seen in Fig. 3. This is illustrated in Fig. 5.

In the next section we turn to a different mechanism of post inhibitory rebound that relies on a novel ionic current, not present in the Hodgkin-Huxley model.

\section{A REBOUND CURRENT}

The response properties of thalamocortical relay neurons are greatly influenced by a low-threshold, transient $\mathrm{Ca}^{2+}$ conductance known as $I_{T}$. When this conductance is evoked, $\mathrm{Ca}^{2+}$ entering the neuron via T-type $\mathrm{Ca}^{2+}$ channels causes a large voltage depolarization known as the low-threshold $\mathrm{Ca}^{2+}$ spike (LTS). Conventional action potentials mediated by fast $\mathrm{Na}^{+}$and $\mathrm{K}^{+}$(delayed-rectifier) currents often ride on the crest of an LTS resulting in a burst response (i.e., a tight cluster of spikes). When a thalamocortical relay neuron is

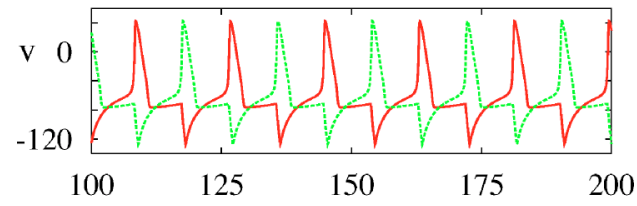

(a)

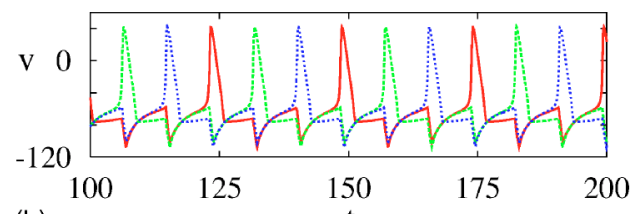

(b)

$\mathrm{t}$

FIG. 5. (Color online) Direct numerical simulations of a network of $N=120$ Hodgkin-Huxley neurons, showing the coexistence of a 2-cluster (top) and a 3-cluster (bottom) state for $\alpha=1.25$ and $J=200$. In the top figure the network has split into two clusters that oscillate in antiphase. In the bottom figure the network has split into three equal sized groups, such that the phase difference between clusters is uniformly distributed on the circle.

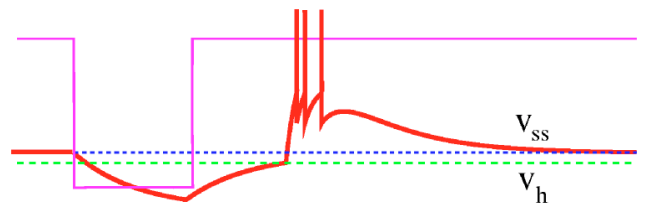

FIG. 6. (Color online) Response of an IFB neuron to a hyperpolarizing current step. Note that upon release from inhibition there is a post inhibitory rebound response consisting of a burst of spikes. Here $v_{\mathrm{ss}}$ denotes the steady state of the neuron.

depolarized (above roughly $-60 \mathrm{mV}$ ), the low-threshold $\mathrm{Ca}^{2+}$ current inactivates with a time constant of $\sim 20 \mathrm{~ms}$. In this situation, further depolarization of sufficient magnitude will evoke a train of action potentials (tonic firing) that is independent of $I_{T}$. However, when a relay neuron is hyperpolarized (below roughly $-65 \mathrm{mV}$ ), the low-threshold current de-inactivates with a time constant of $\sim 100 \mathrm{~ms}$. In this situation release from inhibition results in a post inhibitory rebound response consisting of an LTS and a cluster of 2-10 spikes. A minimal model of this process has been developed by Smith et al. [20] based around intracellular recordings of relay neuron responses to sinusoidal current injection. This minimal "integrate-and-fire-or-burst" (IFB) model was constructed by adding a slow variable (representing the deinactivation level of $I_{T}$ ) to a classical leaky integrate-and-fire (IF) neuron model [21] and is able to quantitatively reproduces salient features of relay neuron response properties in both burst and tonic modes [20,22].

In more detail the IFB model is given by

$$
\begin{gathered}
C \dot{v}=-g_{L}\left(v-v_{L}\right)-g_{T}\left(v-v_{T}\right) h \Theta\left(v-v_{h}\right)+I, \\
\dot{h}= \begin{cases}-h / \tau_{h}^{-} & v \geqslant v_{h}, \\
(1-h) / \tau_{h}^{+} & v<v_{h} .\end{cases}
\end{gathered}
$$

The voltage variable is subject to reset, $\lim _{\delta \rightarrow 0^{+}} v\left(T^{n}+\delta\right)$ $=v_{\text {reset }}$, and refractoriness

$$
T^{n}=\inf \left\{t \mid v(t) \geqslant v_{\theta} ; t \geqslant T^{n-1}+\tau_{R}\right\},
$$

where $v_{\theta}$ is the firing threshold and $\tau_{R}$ is recognized as an absolute refractory period. Here, $g_{L}$ is a constant leakage conductance and $v_{L}$ the leakage reversal potential. The lowthreshold $\mathrm{Ca}^{2+}$ current is given by $I_{T}=g_{T}\left(v-v_{T}\right) h \Theta\left(v-v_{h}\right)$. The slow variable $h$ represents the de-inactivation of the lowthreshold $\mathrm{Ca}^{2+}$ conductance. All parameter values for the IFB model may be found in the Appendix. An example of the response of this model neuron to an inhibitory step input is shown in Fig. 6. This nicely illustrates the sort of rebound response that can be elicited upon release from inhibition. Such a response can only occur if the duration of inhibition is sufficiently long and its strength sufficiently great.

Once again we wish to probe the conditions for a network of neurons to support clustered states, using straightforward mathematical analysis. Since IFB neurons possess an idealized version of the slow T-type calcium current such an analysis naturally complements existing numerical studies of more detailed biophysical networks. In particular we are thinking of the work of Golomb and Rinzel [10], who inves- 
tigate cluster states with a mixture of numerical simulations and Floquet theory. Although the networks they investigate are not ideally suited to a mathematical study, important progress in this direction has been made by Rubin and Terman [23]. These authors consider an idealized model of a neuron with a generic rebound current and show how a network with global coupling may be analyzed using techniques from geometric singular perturbation theory. Although we have had success with a geometric approach in the last section we shall now show how the IFB model is exactly soluble, under some reasonable assumptions, so that we do not have to work in some singular limit. In fact we will explicitly construct cluster states in the limit of slow synaptic responses. However, unlike the last section we will be able to work with both shunts and an arbitrary shape for the postsynaptic conductance.

For postsynaptic currents of the form (10) and (11) which are determined in terms of a set of spike times, there is a simple way to swap to a firing rate description if the synaptic interactions are slow. Since this is already a necessary condition for post inhibitory rebound within the IFB framework we work under this assumption and write the synaptic conductance at the $i$ th neuron in a globally coupled network as

$$
u_{i}(t)=\frac{1}{N} \sum_{j=1}^{N} \int_{0}^{\infty} \eta\left(t^{\prime}\right) f\left(v_{j}\left(t-t^{\prime}\right)\right) \mathrm{dt}^{\prime} .
$$

A more detailed discussion of the derivation of this model can be found in [24], suffice to say that $f(v)$ is to be interpreted as the firing rate of an IFB neuron and $v_{j}=v\left(h_{j}, u_{j}\right)$ is the steady state of the $j$ th presynaptic neuron given by

$$
v(h, u)=\frac{g_{L} v_{L}+g_{T} v_{T} h s+g_{s} v_{s} u}{g_{L}+g_{T} h s+g_{s} u} .
$$

The variable $s$ is a switch such that $s=1$ if $v(h, u)$ crosses $v_{h}$ from below and $s=0$ if $v$ crosses $v_{h}$ from above. If the firing rate is dominated by the refractory mechanism then it is natural to take the instantaneous firing rate as $f(v)=\tau_{R}^{-1} \Theta(v$ $\left.-v_{\theta}\right)$. It is in this case that the model admits to an exact solution.

Generalizing the choice of section II we shall consider the case that $\eta$ is the Green's function of a differential operator $L$ :

$$
L \eta(t)=g_{s} \delta(t)
$$

The equations of motion for the conductances then take the differential form

$$
L u_{i}=\frac{g_{s}}{N} \sum_{j} f\left(v_{j}\right) .
$$

The HFP of the system is given by $u=g_{s} f(v)$ with $h=0$, while the HLC satisfies $L u(t)=g_{s} f(v(t))$. More general $M$-cluster splay states are described by

$$
L u_{i}=\frac{g_{s}}{M} \sum_{k=1}^{M} f\left(v_{k}\right), \quad i=1, \ldots, M
$$

where $u_{i}$ now represents the dynamics of one element in a synchronized cluster. We shall focus on the particular case that $\eta(t)$ is a so-called alpha-function $\eta(t)=\alpha^{2} t^{-\alpha t}$, so that

$$
L=\left(1+\alpha^{-1} \partial_{t}\right)^{2} .
$$

We first consider the construction of the HLC. This can be done by considering a closed orbit of period $\Delta$ and parameterizing the solution in terms of $\Delta$, the time spent above the rebound threshold $v_{h}, \Delta^{+}$, and the time spent above the firing threshold $v_{\theta}, \Delta_{\theta}$. For convenience we choose an origin of time such that at $t=0 v$ crosses $v_{h}$ from below. Assuming that only the most recent burst is influential the HLC takes the explicit form $u(t)=g_{s} Q\left(t, \min \left(t, \Delta_{\theta}\right)\right) / \tau_{R}$, where

$Q(t, a)=\int_{0}^{a} \eta\left(t-t^{\prime}\right) \mathrm{d} t^{\prime}=\mathrm{e}^{-\alpha(t-a)}[1+\alpha(t-a)]-\mathrm{e}^{-\alpha t}[1+\alpha t]$,

and

$$
h(t)= \begin{cases}\bar{h} e^{-\mathrm{t} / \tau_{h}^{-}} & 0 \leqslant \mathrm{t} \leqslant \Delta^{+} \\ \bar{h} e^{-\Delta^{+} / \tau_{h}^{-}} e^{-\left(\mathrm{t}-\Delta^{+}\right) / \tau_{h}^{+}} & \\ +1-e^{-\left(t-\Delta^{+}\right) / \tau_{h}^{+}} & \Delta^{+}<\mathrm{t}<\Delta,\end{cases}
$$

with

$$
\bar{h}=\frac{1-\mathrm{e}^{-\left(\Delta-\Delta^{+}\right) / \tau_{\mathrm{h}}^{+}}}{1-\mathrm{e}^{-\Delta^{+} / \tau_{h}^{-}} \mathrm{e}^{-\left(\Delta-\Delta^{+}\right) / \tau_{h}^{+}}} .
$$

Note that outside their natural domains we periodically extend $u(t)$ and $h(t)$. The three unknowns $\Delta, \Delta^{+}, \Delta_{\theta}$ may then be found by the simultaneous solution of the three equations $v\left(\Delta_{\theta}\right)=v_{\theta}, \quad v\left(\Delta^{+}\right)=v_{h}$, and $v(\Delta)=v_{h}\left(\Delta>\Delta^{+}\right)$. Here, $v(t)$ $=v(h(t), u(t))$ using (22) and $s=1$ for $t \in\left[0, \Delta^{+}\right]$and is zero otherwise. In Fig. 7 we plot the results of such a calculation. This compares extremely well with results obtained from direct numerical simulation. In Fig. 8 we show a plot of activity for a network of size $N=100$, which illustrates the rapid approach of random initial data to the HLC (for the same parameters as in Fig. 7). Figure 9 shows the time evolution of just one of the neurons in the network.

Further numerical simulations of this model with varying $\alpha$ and $g_{s}$ show that the three most common attractors seem to be the HFP, HLC, and 2-cluster state. This is not to say that larger $M$-cluster states do not exist or are not stable, but rather that they may have relatively small basins of attraction. This observation has already been made by Golomb and Rinzel in their studies of more detailed biophysical networks (with slow T-type calcium currents). So although it is easy to generalize the calculation of the HLC to M-cluster splay states by writing $u_{i}(t)=u(t-i \Delta / M)$ for $i=0, \ldots, M-1$ with $h(t)$ given by $(28)$ and 


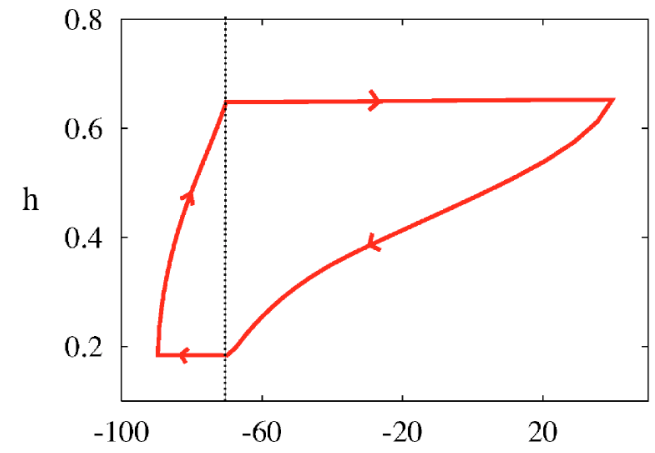

(a)

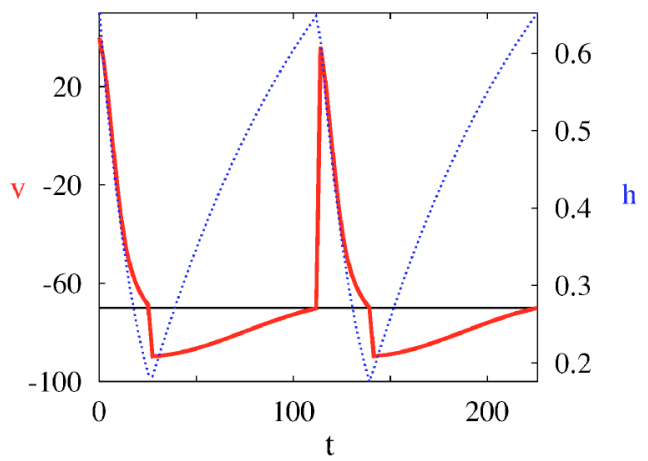

FIG. 7. (Color online) A plot of the explicit HLC solution with $\alpha=0.05, g_{s}=2.0$, and $v_{s}=-100$. For these parameters we find $\Delta$ $=112.9, \Delta^{+}=26.4$, and $\Delta_{\theta}=11.5$.

$$
u(t)=\frac{1}{M} \frac{g_{s}}{\tau_{R}} \sum_{k=0}^{M-1} Q\left(t-k \Delta / M, \min \left(t-k \Delta / M, \Delta_{\theta}\right),\right.
$$

we shall only focus on the case $M=2$ for the above reason. As before solutions are determined in a self-consistent fashion by demanding $v\left(\Delta_{\theta}\right)=v_{\theta}, v\left(\Delta^{+}\right)=v_{h}$ and $v(\Delta)=v_{h}$. A plot of a 2-cluster state is shown in Fig. 10. Once again there is excellent agreement with direct numerical simulations, which we illustrate with the aid of Fig. 11.

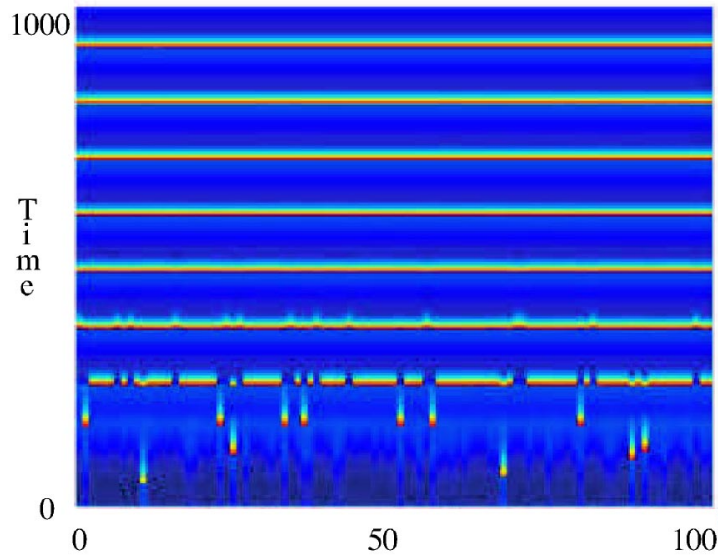

FIG. 8. (Color online) A plot of the voltage for a network of $N=100$ neurons. Bright colors denote high activity and dull colors low activity. All parameters as in Fig. 7. Note the rapid approach to a HLC from random initial data.

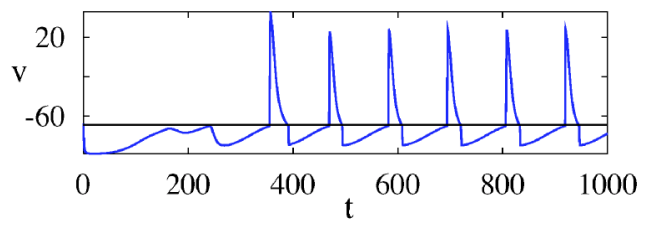

FIG. 9. (Color online) Voltage trace for one of the neurons in Fig. 8, showing good asymptotic agreement with the analytically calculated orbit of Fig. 7.

To establish the stability of the $M$-cluster states one could pursue the approach of Golomb and Rinzel [10] and apply Floquet theory. Indeed having the periodic orbits in closed form is an added bonus for such an approach, as in many models they would only be available numerically. However, we make the important observation that M-cluster states come in pairs, with solutions annihilating in a saddle-node bifurcation under variation of system parameters. Hence, by tracking around these saddle-node bifurcations in parameter space we may determine the existence regions for stable M-cluster states. Within these regions a stable and unstable M-cluster state would co-exist. The result of such a calculation is shown in Fig. 12. This clearly highlights the fact that for slow synapses and sufficiently strong coupling the (stable) HFP, HLC, and 2-cluster state can coexist. With increasing $\alpha$ the system can no longer support a HLC and prefers a 2-cluster state, although with further increase in $\alpha$ only the HFP is found. The borders of existence were found to agree extremely well with direct numerical simulations, although in practice is was hard to find examples (starting from random initial data) of a 2-cluster state coexisting with a HLC.

\section{DISCUSSION}

In this paper we have considered clustering in globally coupled networks of nonoscillatory neurons with inhibitory synaptic connections. A generic mechanism for the generation of such rhythms is that of postinhibitory rebound. To distinguish between the effects of anode break excitation and rebound currents we have analyzed a mathematically tractable neuron model from each of these two classes. For an-

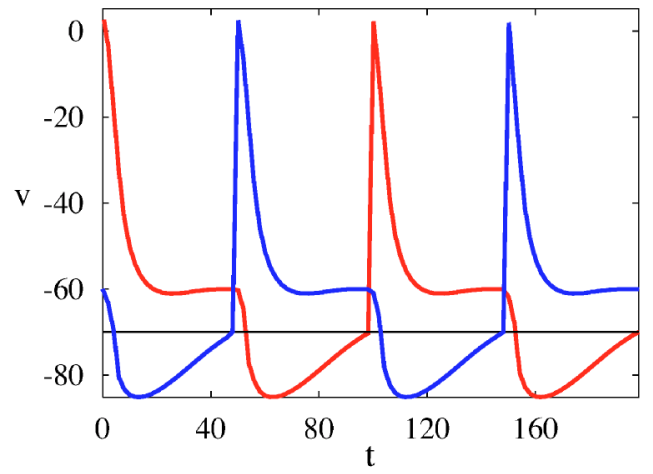

FIG. 10. (Color online) A plot of the 2-cluster state with $\alpha$ $=0.1$. Other parameters as in Fig. 7. For these parameters we find $\Delta=99.1, \Delta^{+}=53.7$, and $\Delta_{\theta}=6.4$. 


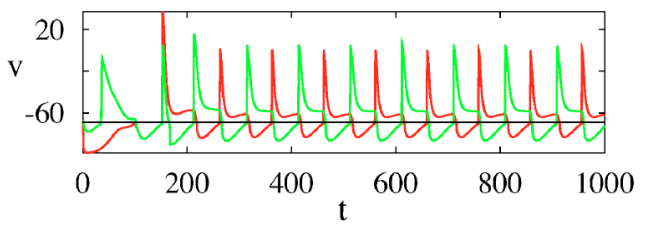

FIG. 11. (Color online) A plot of the voltage for two neurons in a network of $N=100$ neurons. All parameters as in Fig. 10. Note the rapid approach to a 2-cluster state from random initial data, showing good asymptotic agreement with the analytically calculated orbit shown in Fig. 10.

ode break excitation we considered a reduction of the Hodgkin-Huxley model to a form that we have identified as a modified McKean model. Analysis of cluster states was performed in some singular limit (where there is a separation of time scales). Regions for the existence of splay cluster states as a function of synaptic speed and strength were found to be in good qualitative agreement with the full Hodgkin-Huxley model (away from the singular limit). The main conclusion being that slow synapses and weak coupling (above some cut-off) favor small numbers of clusters, while an increase in speed and strength favors larger numbers of clusters. For the analysis of a neuron with an explicit ionic rebound current we chose the IFB model. For the case of slow synapses and a firing rate response dominated by a refractory process we have shown how to construct splay cluster states for a broad class of synaptic shunting models. Direct numerical simulations show that, in contrast to the mechanism of anode break excitation, either a coherent global oscillation or an antiphase rhythm is preferred. One major similarity between the two mechanisms is that the single cluster state is generated for very slow synapses (for some sufficiently strong coupling). Although, for simplicity, we have focused on the construction of the most symmetric cluster states (splay clusters), the techniques we have described are ideally suited for the study of less symmetric states and even partially clustered states. Because of the underlying simplicity of the models we have developed it is also possible to pass over to the case of structured interactions. The results of such an analysis will be presented elsewhere.

For both mechanisms it is also an interesting issue as to whether cluster states are robust to noise. For networks utilizing anode break excitation as the means to generate rhythms this has been explored in a previous paper [25]. Here it was shown that the effect of weak additive Gaussian noise is basically twofold: (i) causing a neuron to switch between different clusters, and (ii) causing the whole system to switch between different cluster states if the system is inside a multistable regime. Moreover, for large networks and moderate noise, it is possible for the system to support a form of coherence resonance [26] (whereby a rhythm is noise induced and would be absent without noise). Similar effects have been observed in model networks with a slow T-type calcium current $[10,27]$. In an extension of their original work on clustering, Golomb and Rinzel [28] have also numerically explored the issue of heterogeneity and found both partially synchronized and partially antisynchronized

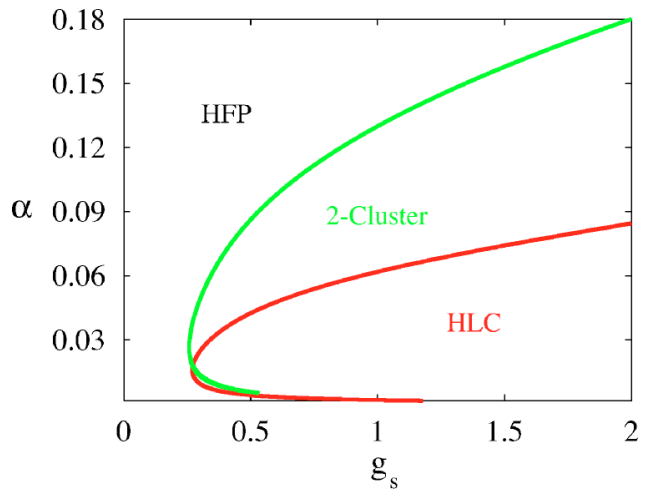

FIG. 12. (Color online) Parameter borders encompassing regions of stable HLC and 2-cluster states in the $\left(\alpha, g_{s}\right)$ plane. Other parameters as in Fig. 7. Note that the HFP is stable everywhere.

states. It remains an open problem to extend the techniques of this paper to heterogeneous systems.

\section{ACKNOWLEDGMENTS}

S.C. was financially supported by the EPSRC through Grant No. GR/R76219.

\section{APPENDIX}

For the Hodgkin-Huxley mode the six functions $\tau_{X}(v)$ and $X_{\infty}(v), X \in\{m, n, h\}$, are obtained from fits with experimental data. It is common practice to write

$$
\tau_{X}(v)=\frac{1}{\alpha_{X}(v)+\beta_{X}(v)}, \quad X_{\infty}(v)=\alpha_{X}(v) \tau_{X}(v),
$$

for $X \in\{m, n, h\}$ where

$$
\begin{gathered}
\alpha_{m}(v)=\frac{0.1(v+40)}{1-\exp [-0.1(v+40)]}, \\
\alpha_{h}(v)=0.07 \exp [-0.05(v+65)], \\
\alpha_{n}(v)=\frac{0.01(v+55)}{1-\exp [-0.1(v+55)]}, \\
\beta_{m}(v)=4.0 \exp [-0.0556(v+65)], \\
\beta_{h}(v)=\frac{1}{1+\exp [-0.1(v+35)]}, \\
\beta_{n}(v)=0.125 \exp [-0.0125(v+65)] .
\end{gathered}
$$

All potentials are measured in $\mathrm{mV}$, all times in $\mathrm{ms}$ and all currents in $\mu \mathrm{A}$ per $\mathrm{cm}^{2}$. We use the following parameter values: $\mu=1 \mu \mathrm{F} \mathrm{cm} \mathrm{cm}^{2}, g_{L}=0.3, g_{K}=36, g_{\mathrm{Na}}=120, V_{L}=-54.402$, $V_{\mathrm{K}}=-77$, and $V_{\mathrm{Na}}=50$.

For the IFB model we use the parameter set $v_{\theta}$ $=-35 \mathrm{mV}, \quad C=0.2 \mu \mathrm{F} / \mathrm{cm}^{2}, \quad g_{L}=0.0354 \mathrm{mS} / \mathrm{cm}^{2}, \quad v_{\text {reset }}$ $=-50 \mathrm{mV}, \quad v_{h}=-70 \mathrm{mV}, \quad \tau_{h}^{-}=20 \mathrm{~ms}, \quad \tau_{h}^{+}=100 \mathrm{~ms}, \quad g_{T}$ $=0.07 \mathrm{mS} / \mathrm{cm}^{2}, v_{T}=120 \mathrm{mV}$, and $\tau_{R}=5 \mathrm{~ms}$. 
[1] R. A. Satterlie, Science 229, 402 (1985).

[2] E. A. Arbas and R. L. Calabrese, J. Neurosci. 7, 3953 (1987).

[3] A. Roberts, Sci. Prog. 74, 31 (1990).

[4] Y. I. Arshavsky, G. N. Orlovsky, Y. V. Panchin, A. Roberts, and S. R. Soffe, Trends Neurosci. 16, 227 (1993).

[5] E. W. Large and J. D. Crawford, J. Comput. Neurosci. 13, 125 (2002).

[6] D. H. Perkel and B. Mulloney, Science 185, 181 (1974).

[7] T. G. Brown, J. Physiol. (London) 48, 18 (1914).

[8] J. D. Angstadt and R. L. Calabrese, J. Neurosci. 9, 2846 (1989).

[9] E. A. Arbas and R. L. Calabrese, J. Neurosci. 7, 3945 (1987).

[10] D. Golomb and J. Rinzel, Physica D 72, 259 (1994).

[11] M. Steriade, E. G. Jones, and R. R. Linas, Thalamic Oscillations and Signalling (J. Wiley, New York, 1990).

[12] D. Golomb, D. Hansel, B. Shraiman, and H. Sompolinsky, Phys. Rev. A 45, 3516 (1992).

[13] D. Hansel, G. Mato, and C. Meunier, Phys. Rev. E 48, 3470 (1993).

[14] K. Okuda, Physica D 63, 424 (1993).

[15] H. P. McKean, Adv. Math. 4, 209 (1970).

[16] A. L. Hodgkin and A. F. Huxley, J. Physiol. (London) 117,
500 (1952).

[17] L. F. Abbott and T. B. Kepler, in Statistical Mechanics of Neural Networks, Lecture Notes in Physics, edited by L. Garrido (Springer-Verlag, Berlin, 1990), Vol. 368, pp. 5-18.

[18] S. Coombes, Physica D 2820, 1 (2001).

[19] M. Denman-Johnson and S. Coombes, Phys. Rev. E 67, 051903 (2003).

[20] G. D. Smith, C. L. Cox, S. M. Sherman, and J. Rinzel, J. Neurophysiol. 83, 588 (2000).

[21] B. W. Knight, J. Gen. Physiol. 59, 734 (1972).

[22] S. Coombes, M. R. Owen, and G. D. Smith, Phys. Rev. E 64, 041914 (2001).

[23] J. Rubin and D. Terman, J. Math. Biol. 41, 513 (2000).

[24] S. Coombes, Phys. Rev. E 67, 041910 (2003).

[25] D. T. W. Chik and Z. D. Wang, Phys. Rev. E 68, 031907 (2003).

[26] G. Hu, T. Ditzinger, C. N. Ning, and H. Haken, Phys. Rev. Lett. 71, 807 (1993).

[27] P. H. E. Tiesinga and J. V. Jose, J. Comput. Neurosci. 9, 49 (2000).

[28] D. Golomb and J. Rinzel, Phys. Rev. E 48, 4810 (1993). 